\title{
Migration Between Two Inverse Time-Overcurrent Curves of Different Standard: Experience at Siguragura Power Station
}

\author{
Bagus B Gautama \\ Department of Maintenance and Engineering, PT Indonesia Asahan Aluminium (Persero) \\ bagusbpg@inalum.id
}

\begin{abstract}
The development in electrical protection relay technology has encouraged inevitable replacement of electromechanical protection relays with microprocessor-based protection relays recently developed. Some problems occurred, especially during the replacement program. The most challenging one is the migration from a manufacturer with non-standard inverse time-overcurrent curve to IEC or IEEE curves, especially when both microprocessor-based and electromechanical protection relays are installed in the same system. A study of protection coordination for the overall system prior to the replacement program is needed. However, this exhaustive approach can be avoided by emulating existing non-standard inverse time overcurrent curve on the new microprocessor-based protection relay, as long as such curve is still adequate to be re-implemented. This paper aims to develop a general procedure to emulate electromechanical protection relay on microprocessor-based protection relay. It is done by tuning the time multiplier setting of the standard curve to match data point of current and time from the non-standard curve. This procedure has been tested with great success during the renewal of feeder protection relay at Siguragura Power Station. It is concluded that a particular non-standard characteristics curve of electromechanical protection relay can be consistently replaced by a certain standard curve of microprocessor-based protection relay.
\end{abstract}

Keywords : Inverse time-overcurrent curve, electro-mechanical protection relay, microprocessor-based protection relay and nonlinear curve fitting

\section{Introduction}

Electric power stations and facilities built around 1970s and 1980s, such as Siguragura Power Station, have employed predominantly electromechanical (also known as induction-disk) protection relays as the main component of generator, transformer, bus bar, and feeder protection systems-with only small part of the system used solid-state protection relays. Those electromechanical protection relays are widely known for having excellent reliability even after more than 30 years of service [1]. With carefullyplanned periodic maintenance, their service life may even be extended up to 20 years more.

However, electromechanical protection relays are also subjected to the same problem encountered by any other equipment: they will eventually fail, with common mode of failure includes unrepairable friction, oxidation and outgassing of material, deteriorated wire insulation, presence of metallic whiskers, etc. [2]. Plant engineer must arrange a replacement program for the existing deteriorated relays.

With proven reliability and absence of any serious disadvantage, it is not uncommon that some plant engineers are actually still inclined to reapply this kind of protective device in their proposal of replacement or refurbishment program. On the other hand, manufacturers of electrical protection relay have clearly stated that the production line of electromechanical protection relay has been discontinued and that they have been solely manufacturing microprocessor-based protection relay. Beginning around 1990s, microprocessor-based protection relay has gained so much popularity with its new features of monitoring, control, disturbance and event handling, communication, beside its main feature of protection [3,4]. Albeit there is still heated debate on the advertised superiority of microprocessor-based protection relay over its electromechanical counterpart [5], plant engineers thus have no alternative other than accepting this shift in technology [1].

During the implementation of replacement program, the process is not as simple as it may seem. First, microprocessor-based protection relay uses standardized characteristics, mostly 
conforming to IEC 60255-151 or IEEE C37.112$1996[6,7]$, while the characteristics of electromechanical relay is specially designed by its manufacturer, such as in [8, 9]. Besides, considering the continuity of plant operation, replacement program is most often executed in several stages, instead of one-continuous project, giving rise to an unavoidable condition of mixed presence of both microprocessor-based and electromagnetic protection relay in the system, although temporarily. Thus, the introduction of microprocessor-based protection relay into existing system requires careful calculation to ensure that protection coordination is always fulfilled.

\section{Theoretical Review}

The coordination of protective devices should be calculated after load-flow and short-circuit studies, based on equipment specifications and the topology of the electrical network as well as its critical operating scenarios [10]. Unless no change has been made on the topology and operating scenario of electrical network, the previous loadflow and short-circuit studies are considered still valid, the ones used earlier as the basis to set electromechanical protection relay in the first place. Thus, existing pick up current setting need no revision, whereas the selection of inverse timeovercurrent curve and time multiplier setting will be restudied such that it coordinates well with downstream and upstream protection relays.

The mixed presence of electromechanical and microprocessor-based protection relays in the system, each having different shape of timeovercurrent curve, makes coordination study more difficult [10]. Fortunately, most microprocessorbased protection relay has characteristics defined by mathematical equation, making it possible to emulate the characteristics of electromechanical protection relay [11], using various approaches as described in [12]. This clearly gives a much simpler solution to our protection coordination problem.

In this paper, an alternative approach to emulate the electromechanical protection relay is presented, i.e. by utilizing the built-in standardized characteristics curves of micropro-cessor-based protection relay instead of creating a new special curve. This is done first by finely tuning the time multiplier setting on each standardized characteristics curve such that it gets as closely as possible to the known data points from previous non-standard characteristics curve. Then, among these tuned curves, plant engineer must select the most appropriate one(s). Finally, the new chosen curve(s) must be graphed together with upstream and downstream curves to confirm that the required coordination had been successfully achieved.

Now, let us consider microprocessor-based protection relay having built in general characteristics curve as follows,

$$
t=T_{\mathrm{MS}}\left(\mathrm{A}+\frac{\mathrm{B}}{M^{\mathrm{C}}-1}\right)
$$

where $t$ is operating time, $T_{\mathrm{MS}}$ is time multiplier setting, $M$ is multiple of pickup current setting, while $\mathrm{A}, \mathrm{B}$, and $\mathrm{C}$ are constants defining curve type as shown in Table 1.

Table 1. Constants of characteristics curve $[6,7]$

\begin{tabular}{|c|c|c|c|}
\hline Curve Type & $\mathrm{A}$ & $\mathrm{B}$ & $\mathrm{C}$ \\
\hline US Moderately Inverse & 0.0226 & 0.0104 & 0.02 \\
US Inverse & 0.180 & 5.95 & 2 \\
US Very Inverse & 0.0963 & 3.88 & 2 \\
US Extremely Inverse & 0.0352 & 5.67 & 2 \\
US Short-Time Inverse & 0.00262 & 0.00342 & 0.02 \\
IEC Standard Inverse & 0 & 0.14 & 0.02 \\
IEC Very Inverse & 0 & 13.5 & 1 \\
IEC Extremely Inverse & 0 & 80 & 2 \\
IEC Long-Time Inverse & 0 & 120 & 1 \\
IEC Short-Time Inverse & 0 & 0.05 & 0.04 \\
IEEE Moderately Inverse & 0.114 & 0.0515 & 0.02 \\
IEEE Very Inverse & 0.491 & 19.61 & 2 \\
IEEE Extremely Inverse & 0.1217 & 28.2 & 2 \\
\hline
\end{tabular}

To tune characteristics curve, the method of nonlinear curve fitting will be used. This method aims to find the best-fitting curve to a given set of points by evaluating the sum of the squares of the offsets of the points from the curve, mathematically denoted as $R^{2}$ [13]. In our case, given paired data point of time $t_{j}$ and current $M_{j}$ from old characteristics curve,

$$
\begin{aligned}
t_{j} & =\left\{t_{1}, t_{2}, \cdots, t_{n}\right\} \\
M_{j} & =\left\{I_{1}, I_{2}, \cdots, I_{n}\right\}
\end{aligned}
$$

the objective is to find $T_{\mathrm{MS}}$ such that $R^{2}$ becomes minimum, where

$$
R^{2}=\sum_{j=1}^{n}\left[t_{j}-T_{\mathrm{MS}}\left(\mathrm{A}+\frac{\mathrm{B}}{M_{j}^{\mathrm{C}}-1}\right)\right]^{2}
$$

Those data point can be collected by testing the relay directly or evaluating its time-overcurrent curves. Plant engineer then chooses the lowest value of $R^{2}$, which indicates that the corresponding 
curve most resembles the non-standard characteristics curve to be replaced.

Microsoft Excel $®$ Solver add-in will be used to solve equation (3) with the general procedure as described in [14].

In addition, Pearson correlation coefficient will be calculated to evaluate the linear correlation between the tuned curve and collected data point.

\section{Research Methodology}

To put this alternative approach into a test, we will consider and analyze a case encountered during first stage replacement program of protection relay for $6.6 \mathrm{kV}$ network at Siguragura Power Station as shown in Figure 1 below, with corresponding coordination graph as shown in Figure 2. In this first stage, Relay2 was replaced, i.e. from electromechanical protection relay Meidensha Electric's KIO-CR to microprocessorbased one SEL 351A.

The required data point of time and current for existing Relay 2 are given in Table 2 . Those data point are acquired from characteristics curve available in catalogue [8] and some of them have been verified by measurement. Later, they will be used to tune characteristics curve of SEL 351A by nonlinear curve fitting method as described in Section 2.

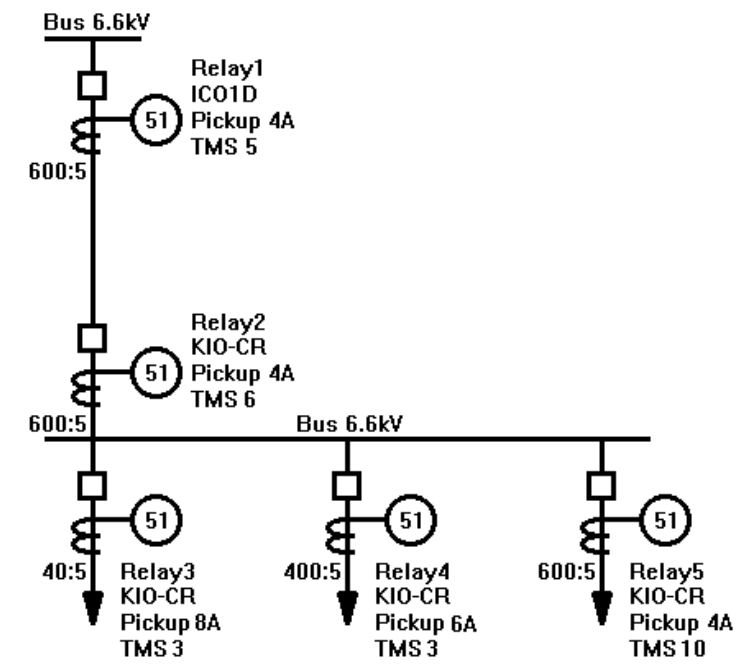

Figure 1. Case study for replacement program of protection relay for $6.6 \mathrm{kV}$ network at Siguragura Power Station.

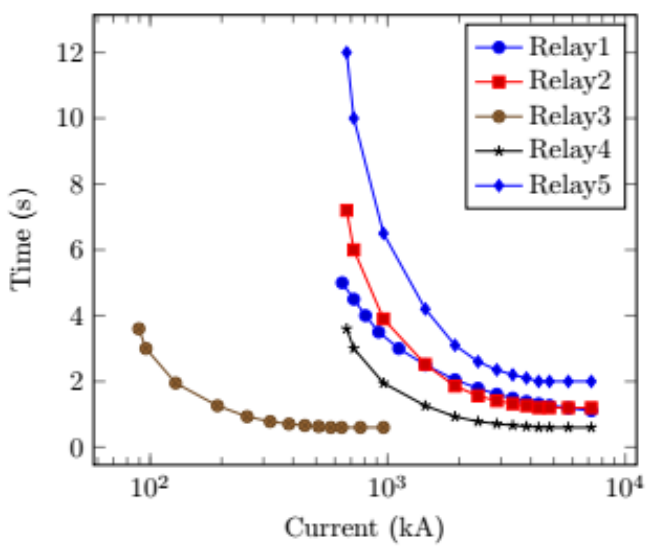

Figure 2. Coordination graph for network in Figure 1.

Table 2. Current Versus Time for Existing Relay2

\begin{tabular}{|c|c|c|}
\hline Current (A) & $\begin{array}{c}\text { Multiple of } \\
\text { Pickup Current }\end{array}$ & Time (s) \\
\hline 5.60 & 1.40 & 7.20 \\
6.00 & 1.50 & 6.00 \\
8.00 & 2.00 & 3.90 \\
12.00 & 3.00 & 2.52 \\
16.00 & 4.00 & 1.86 \\
20.00 & 5.00 & 1.56 \\
24.00 & 6.00 & 1.41 \\
28.00 & 7.00 & 1.32 \\
32.00 & 8.00 & 1.26 \\
36.00 & 9.00 & 1.20 \\
40.00 & 10.00 & 1.20 \\
48.00 & 12.00 & 1.20 \\
60.00 & 15.00 & 1.20 \\
\hline
\end{tabular}

Second-stage replacement program is also discussed, in which Relay4 was replaced, to check the validity of the proposed procedure for tuning the characteristics curve, especially for the same relays having different settings. The required data point of time and current for existing Relay 4 are given in Table 3 below, some of them have been verified by measurement as well.

Table 3. Current Versus Time for Existing Relay4

\begin{tabular}{|c|c|c|}
\hline Current (A) & $\begin{array}{c}\text { Multiple of } \\
\text { Pickup Current }\end{array}$ & Time (s) \\
\hline 8.40 & 1.40 & 3.60 \\
9.00 & 1.50 & 3.00 \\
12.00 & 2.00 & 1.95 \\
18.00 & 3.00 & 1.26 \\
24.00 & 4.00 & 0.93 \\
30.00 & 5.00 & 0.78 \\
36.00 & 6.00 & 0.71 \\
42.00 & 7.00 & 0.66 \\
48.00 & 8.00 & 0.63 \\
54.00 & 9.00 & 0.60 \\
60.00 & 10.00 & 0.60 \\
72.00 & 12.00 & 0.60 \\
90.00 & 15.00 & 0.60 \\
\hline
\end{tabular}


Finally, a comparison is also made for the case of replacing Relay1, this time for different set of brands, i.e. from electromechanical protection relay Toshiba's IC01D to microprocessor-based one Toshiba's GRD140. The required data point of time and current for existing Relay1 are given in Table 4 below.

Table 4. Current Versus Time for Existing Relay1

\begin{tabular}{|c|c|c|}
\hline Current (A) & $\begin{array}{c}\text { Multiple of } \\
\text { Pickup Current }\end{array}$ & Time (s) \\
\hline 5.36 & 1.34 & 5.00 \\
6.00 & 1.50 & 4.50 \\
6.72 & 1.68 & 4.00 \\
7.64 & 1.91 & 3.50 \\
9.28 & 2.32 & 3.00 \\
12.00 & 3.00 & 2.50 \\
16.00 & 4.00 & 2.05 \\
20.00 & 5.00 & 1.79 \\
24.00 & 6.00 & 1.61 \\
28.00 & 7.00 & 1.49 \\
32.00 & 8.00 & 1.39 \\
36.00 & 9.00 & 1.32 \\
40.00 & 10.00 & 1.27 \\
48.00 & 12.00 & 1.19 \\
60.00 & 15.00 & 1.11 \\
\hline
\end{tabular}

\section{Results and Discussion}

\subsection{First Stage Replacement Program}

Table 5 shows the calculated value of $R^{2}$ and Pearson correlation coefficient for each curvefitted characteristics of microprocessor-based protection relay using data point of Table 2 . Since SEL protection relays to be used in this case do not originally recognize IEEE curves, no calculation will be performed for such curves.

Table 5. Result of nonlinear curve fitting for the case of replacing Relay2

\begin{tabular}{|c|c|c|c|}
\hline Curve Type & $\begin{array}{c}\text { Calc. } \\
T_{\text {MS }}\end{array}$ & $R^{2}$ & $\begin{array}{c}\text { Pearson } \\
\text { Corrltn. } \\
\text { Coeff. (\%) }\end{array}$ \\
\hline US Moderately Inverse & 4.70 & 0.23522 & 99.82 \\
US Inverse & 1.26 & 12.2562 & 99.12 \\
US Very Inverse & 1.94 & 13.0955 & 99.12 \\
US Extremely Inverse & 1.35 & 16.2804 & 99.12 \\
US Short-Time Inverse & 14.55 & 0.33867 & 99.82 \\
IEC Standard Inverse & 0.36 & 0.41890 & 99.82 \\
IEC Very Inverse & 0.23 & 8.30651 & 99.71 \\
IEC Extremely Inverse & 0.10 & 17.4531 & 99.12 \\
IEC Long-Time Inverse & 0.03 & 8.30651 & 99.71 \\
IEC Short-Time Inverse & 2.02 & 0.47940 & 99.82 \\
\hline
\end{tabular}

Referring to Table 5, there are four curves to be considered as the most appropriate candidates to replace characteristics curve of Relay2 since they have the lowest $R^{2}$, i.e. US Moderately Inverse, US
Short-Time Inverse, IEC Standard Inverse and IEC Short-Time Inverse. These four finely-tuned curves will then be graphed together alongside upstream and downstream curves. This is shown in Figure 3 below.

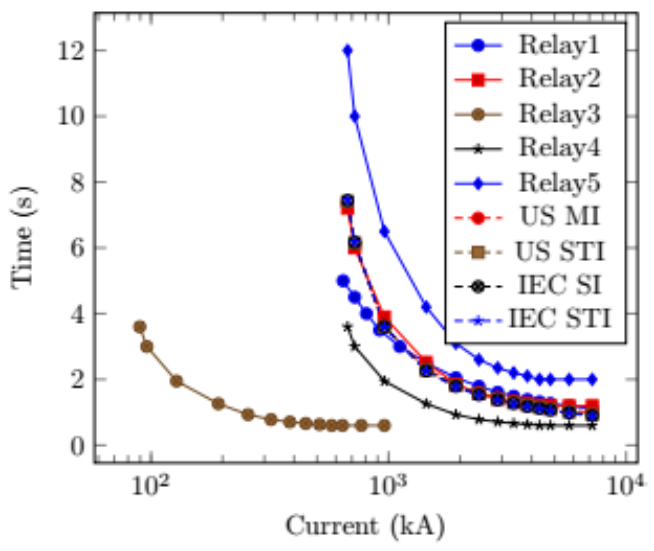

Figure 3. Coordination graph for network in Figure 1 , including four finely-tuned curves.

Judging from Figure 3, these four finely-tuned curves are quite well in emulating the characteristics curve of existing Relay3. Despite such eligibility, it was then decided to select IEC Standard Inverse curve with $T_{\mathrm{MS}} 0.36$ in anticipation of replacing the upstream-side Relay 1 with different brand of protection relay on another stage of replacement program-such mixed presence of various brand of protection relay gets more common today and we will also consider this case later in Section 4.3. This reason seems trivial at first glance. Nevertheless, it is commonly thought that IEC curves are more favorable than US curves, since they are more widely available in many other brands of microprocessor-based protection relay.

\subsection{Second Stage Replacement Program}

Table 6 shows similar data as Table 5, but for the case of replacing Relay4. By following our previous reasoning, it turns out that there are also four curves to be considered as the most appropriate candidates to replace characteristics curve of Relay4. These four curves happen to be exactly the same as in earlier case of replacing Relay2, only that the calculated $T_{\mathrm{MS}}$ becomes smaller, proportional to corresponding time multiplier setting of the replaced relay. In our first case, the old relay's time multiplier setting is 6 and the calculated $T_{\mathrm{MS}}$ of the chosen new relay is 0.36 . Now as the former is divided by two, the latter is 
also divided by two. This suggests, or even proves, that a particular nonstandard characteristics curve can be consistently replaced with certain standardized curve, regardless of time multiplier setting used by corresponding electromechanical protection relay.

Table 6. Current Versus Time for Relay4

\begin{tabular}{|c|c|c|c|}
\hline Curve Type & $\begin{array}{c}\text { Calc. } \\
T_{\text {MS }}\end{array}$ & $R^{2}$ & $\begin{array}{c}\text { Pearson } \\
\text { Corrltn. } \\
\text { Coeff. (\%) }\end{array}$ \\
\hline US Moderately Inverse & 2.35 & 0.05881 & 99.82 \\
US Inverse & 0.63 & 3.06404 & 99.12 \\
US Very Inverse & 0.97 & 3.27388 & 99.12 \\
US Extremely Inverse & 0.67 & 4.07011 & 99.12 \\
US Short-Time Inverse & 7.27 & 0.08467 & 99.82 \\
IEC Standard Inverse & 0.18 & 0.10472 & 99.82 \\
IEC Very Inverse & 0.12 & 2.07663 & 99.71 \\
IEC Extremely Inverse & 0.05 & 4.36328 & 99.12 \\
IEC Long-Time Inverse & 0.01 & 2.07663 & 99.71 \\
IEC Short-Time Inverse & 1.01 & 0.11985 & 99.82 \\
\hline
\end{tabular}

Continuing our analysis, Figure 4 shows the updated coordination graph which incorporates two new microprocessor-based protection relays: Relay2 and Relay4. So far, the required protection coordination has been successfully achieved.

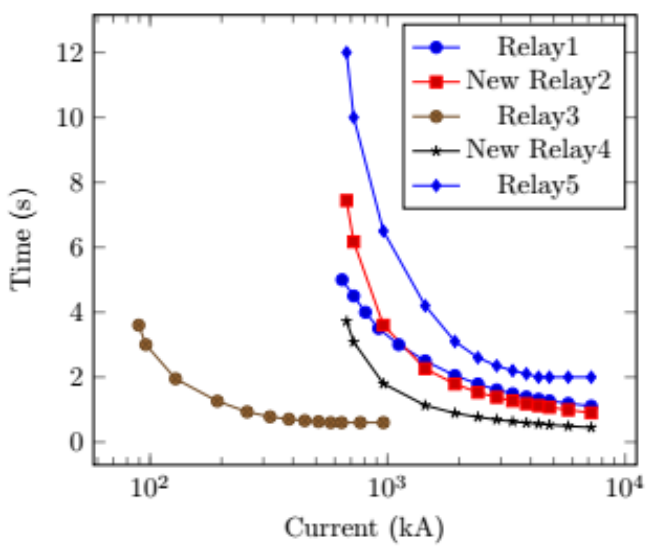

Figure 4. Coordination graph for network in Figure 1, with two new Relay2 and Relay4.

Using the principles of proportionality as discussed earlier, it then becomes possible to deduce that Relay 3 and Relay 5 can be replaced by microprocessor-based protection relay having IEC Standard Inverse characteristics curve with $T_{\mathrm{MS}}$ equal to 0.18 and 0.60 , respectively. Not surprisingly, this indeed turns out to be the correct choice. Figure 5 shows the updated coordination graph and once again meeting the required protection coordination.

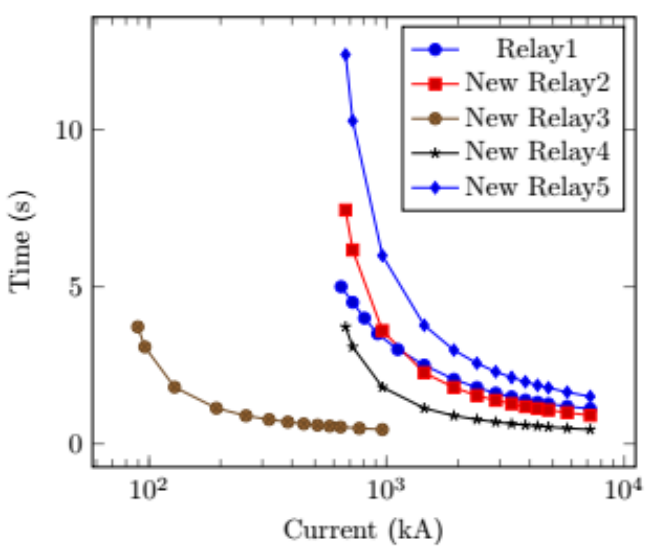

Figure 5. Coordination graph for network in Figure 1, with new Relay2, Relay3, Relay4 and Relay5.

\subsection{Other Stage Replacement Program with Different Brand of Protection Relay}

Table 7 shows calculated value of $R^{2}$ and Pearson correlation coefficient for the case of replacing Relay1. Unlike SEL, Toshiba protection relays do recognize IEEE curves, but not some US curves.

Table 7. Result of nonlinear curve fitting for the case of replacing Relay 1

\begin{tabular}{|c|c|c|c|}
\hline Curve Type & $\begin{array}{c}\text { Calc. } \\
T_{\mathrm{MS}}\end{array}$ & $R^{2}$ & $\begin{array}{c}\text { Pearson } \\
\text { Corrltn. } \\
\text { Coeff. } \\
(\%)\end{array}$ \\
\hline US Inverse & 0.90 & 24.4380 & 93.73 \\
US Short-Time Inverse & 11.07 & 6.10762 & 96.84 \\
IEC Standard Inverse & 0.27 & 6.42000 & 96.84 \\
IEC Very Inverse & 0.17 & 18.6037 & 95.99 \\
IEC Extremely Inverse & 0.07 & 29.4671 & 93.73 \\
IEC Long-Time Inverse & 0.02 & 18.6037 & 95.99 \\
IEEE Moderately Inverse & 0.73 & 5.55747 & 96.84 \\
IEEE Very Inverse & 0.27 & 25.2498 & 93.72 \\
IEEE Extremely Inverse & 0.19 & 28.7020 & 93.72 \\
\hline
\end{tabular}

Referring to Table 7, there are only three curves to be considered as the most appropriate candidates to replace characteristics curve of Relay1, i.e. US Short-Time Inverse, IEC Standard Inverse and IEEE Moderately Inverse. During this separate stage of replacement program with different project executor and, of course, different brand as well, it was again naturally decided to pick IEC Standard Inverse curve due to its universality among brands of microprocessor-based protection relay, the very same reason discussed earlier in Section 4.1. Besides, it is considered advantageous to have same characteristics curve within the network, avoiding crossing point(s) among curves 
which may lead to poor protection coordination. Again, Figure 6 shows the updated coordination graph.

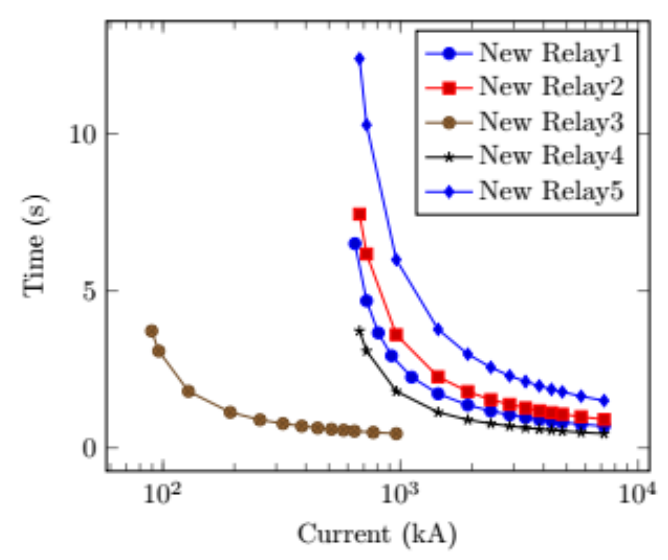

Figure 6. Coordination graph for network in Figure 1, with new Relay1, Relay2, Relay3, Relay4 and Relay5.

According to Figure 6, protection coordination has been improved by getting rid of cross point(s) between old Relay1 and new Relay2. This settings of new microprocessor-based relays are considered adequate for our purpose. Nevertheless, further examination by plant engineer requires that Relay 1 and Relay2 to have closer characteristics curve because electric power on the associated feeder does not always flow forward, but in some rare occasion, it is intentionally reversed. Thus, $T_{\mathrm{MS}}$ of Relay1 is adjusted from 0.27 to 0.40 , making it slightly slower than (or graphically above) Relay2. Figure 7 and Figure 8 shows our final coordination graph and updated specification of protection relay.

\subsection{Accuracy of Standardized Curve in Emulating Non-Standard Curve}

The curve-fitted IEC Standard Inverse is very well in emulating both KIO-CR and IC01D electromechanical protection relays as indicated by Pearson correlation coefficient as high as $\mathbf{9 9 . 8 2 \%}$ and $96.84 \%$, respectively. It means that the exponential form of equation which defines standard characteristics curve are very good in approximating the behavior of electromechanical protection relay. This suggests the high applicability of our proposed procedure.

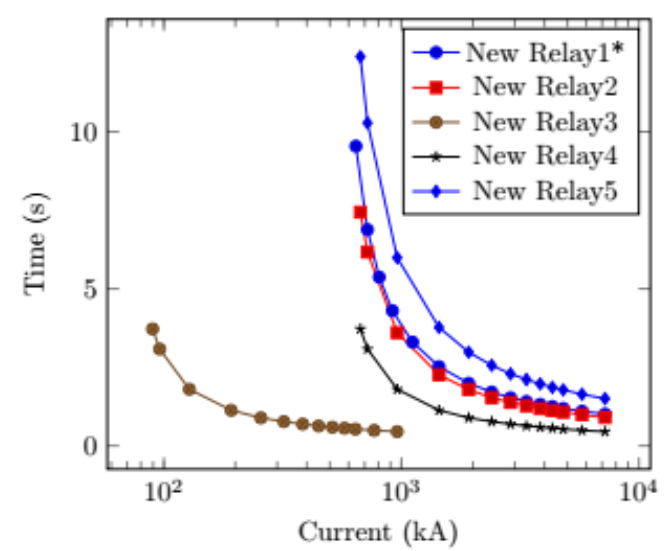

Figure 7. Final coordination graph for network in Figure 1, with new Relay1 (modified), Relay2, Relay3, Relay4 and Relay5.

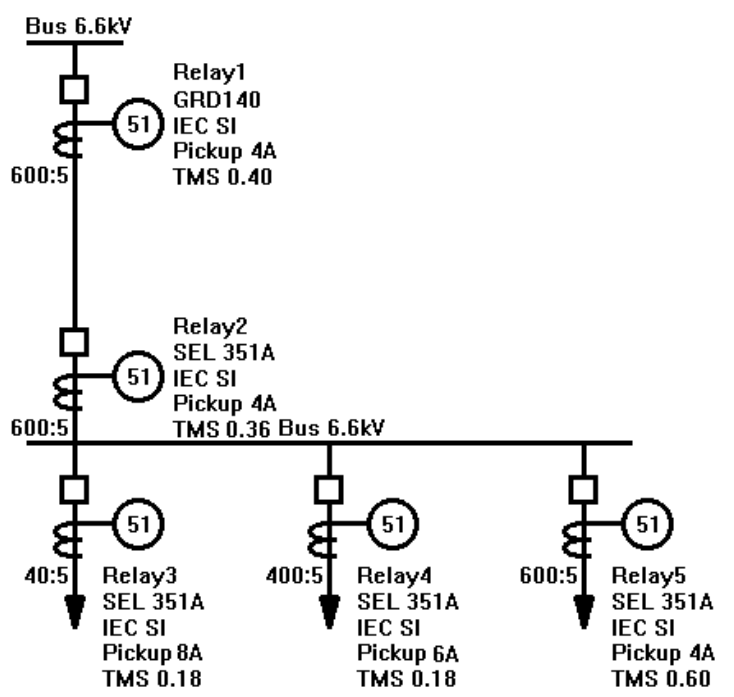

Figure 8. $6.6 \mathrm{kV}$ network at Siguragura Power Station with new microprocessor-based protection relays.

\section{Conclusion}

A general procedure to emulate characteristics of electromechanical protection relay on microprocessor-based protection relay has been proposed using nonlinear curve fitting, in which time multiplier setting of standard characteristics curve is finely tuned based on information from existing nonstandard characteristics curve. This general procedure also has been implemented, with great success, during actual protection relay replacement program, involving various different brands of microprocessor-based protection relay.

Based on experience, a particular nonstandard characteristics curve of electromechanical protection relay can be consistently replaced with certain standardized curve provided by 
microprocessor-based protection relay. The calculated time multiplier setting of new relay is just proportional to existing time multiplier setting of old relay.

\section{References}

[1] V. Gurevich, "Reliability of microprocessor-based protective devices revisited," Journal of Electrical Engineering, vol. 60, no. 5, pp. 1-6, 2009.

[2] G. Goldfarb, "Hard to find maintenance tips for electromechanical relays," in $70^{\text {th }}$ Annual Conference for Protective Relay Engineers, 2017, pp. 1-6.

[3] A. Abdelmoumene and H. Bentarzi, "A review on protective relays' developments and trends," Journal of Energy in Southern Africa, vol. 25, no. 2, pp. 91-95, 2014.

[4] S. A. Wright, et al., "Benefits of upgrading protection schemes for hydroelectric power plants," in IEEE Systems Technical Conference on Industrial and Commercial Power, 2005, pp. 218 - 225.

[5] V. Gurevich, "Microprocessor protection relays: new prospects or new problems?" Electrical Engineering and Electromechanics, no. 3, pp. 18-26, 2006.

[6] Schweitzer Engineering Laboratories, "SEL-351A, -1 protection system instruction manual," pp. 9.5, 2015.

[7] Toshiba Corporation, "Instruction manual directional overcurrent protection relay GRD140," pp. 11, 2004.

[8] Meidensha Electric Corporation, "KIOCR, KIO OR, KIO OSR overcurrent relay," pp. 9-10, 1981.
[9] Toshiba Corporation, "Instructions for overcurrent relay types ICO1D, ICO1E, ICO1F," pp. 5, 1963.

[10] O. A. Soria, et al., "Overcurrent relay with unconventional curves and its application in industrial power systems," Electric Power System Research, vol. 110, pp. 113121, 2014.

[11] K. Zimmerman, "Microprocessor-based distribution relay applications," in American Public Power Association's Engineering \& Operations Workshop, 1996.

[12] F. J. Wu, et al., "Modeling electromechanical overcurrent relays using singular value decomposition," Journal of Applied Mathematics, vol. 2012, pp. 1-18, 2012.

[13] E. W. Weisstein. (2019, 11 April). Least squares fitting. Available: http:// mathworld.wolfram.com/LeastSquaresFitt ing.html/

[14] E. G. John, "Simplified curve fitting using spreadsheed add-ins," Journal of Engineering Education, vol. 14, no. 5, pp. 375-380, 1998.

\section{Author biography}

Bagus B Gautama obtained Bachelor degree in Electrical Engineering from Universitas Gadjah Mada, Yogyakarta (Indonesia) in 2012. Currently, he is maintenance staff at Department of Maintenance and Engineering, Asahan II HydroElectric Power Plant, PT Indonesia Asahan Aluminium (Persero). His main interests include electrical protection, maintenance engineering, power system dynamics and stability, power electronics, and energy management. 